\title{
Patients' Concerns regarding Symptoms Severity and Treatment of Benign Prostate Hypertrophy (BPH)
}

\author{
Amal Samir Ahmed Abdel Maqsoud, Assistant Professor \\ Medical Surgical Nursing, Faculty of Nursing, Alexandria University \\ Abeer Mohamed Nour, Lecturer \\ Medical Surgical Nursing, Faculty of Nursing, Alexandria University
}

\begin{abstract}
Benign prostate hypertrophy $(\mathrm{BPH})$ has an impact on quality of life and symptoms are crucial in causing bother. Therefore a patient's concerns of the disease, and his subjective ranking of therapeutic priorities should be elicited, and the patient's expectancies should be considered. Objective: to describe patients' concerns regarding symptoms severity and treatment of BPH. Setting: The study was carried out at the Urologic Outpatient Department of the Health Insurance Hospital in Alexandria, Egypt. Subjects: A convenience sample of 100 adult male patients diagnosed with benign prostate hypertrophy were recruited to participate in the study. Tools: a structured interview schedule was used in this study including bio-sociodemographic data, the International Prostate Symptom Score (IPSS); additional questions relating to patients' concerns about symptoms severity and treatment of enlarged prostate. Results: The findings indicated that more than half of the patients (52\%) had moderate lower urinary tract symptom severity (LUTS). The main concerns first experienced by patients seeking medical advice for symptoms of BPH were the impact of symptoms on social and professional life, fear of cancer, frustration and embarrassment caused by symptoms. The main concerns of a large number of patients when taking medication were the ability of medication to reduce the risk of surgery and relieve symptoms within few weeks or months. Conclusion: This study provides a valuable insight into the concerns of patients regarding BPH and its management. Moreover, it emphasizes the need for consideration of patient preferences during clinical decision-making. Recommendations: The study recommended that it is vital that health teams assess and fully understand their patients' satisfaction with BPH treatment, their preferred treatment options and expectations
\end{abstract}

Keywords: Patients' concerns; benign prostate hypertrophy.

\section{Introduction}

Benign prostate hypertrophy $(\mathrm{BPH})$ is a common condition affecting men as they age. Previous population-based studies have reported that approximately $30-50 \%$ of men over age 50 have moderate-to-severe lower urinary tract symptoms (LUTS), with the prevalence increasing with age ${ }^{(1)}$. As many as 14 million men in the United States have symptoms of BPH. Worldwide, approximately 30 million men have symptoms related to $\mathrm{BPH}^{(2)}$. $\mathrm{BPH}$ can be defined histologically as a hyperplasia of the stromal and epithelial tissue of the prostate and it can be associated with lower urinary tract symptoms (LUTS), benign prostatic enlargement (BPE) and bladder outlet obstruction (BOO) ${ }^{(3)}$.

The In BPH the prostate enlarges and obstructs the urine flow, causing symptoms collectively known as prostatism hesitancy, intermittency, dribbling, impairment of the force of the stream, and the sensation of incomplete bladder emptying. Symptoms often begin gradually, growing more severe as the bladder muscle loses its ability to overcome resistance to outflow ${ }^{(4)}$. 
Patients with $\mathrm{BPH}$ experience a significant deterioration in quality of life because of their condition, reporting changes in sleep patterns, anxiety and embarrassment, altered mobility, changes in leisure, daily living activities and sexual

activities and unsatisfaction with sexual relationships $^{(3)}$. In some men, BPH progression can lead to complications such as a cute urinary retention, bladder decompensation, recurrent urinary tract infections and the need for prostate surgery ${ }^{(1)}$. In this context, it has been reported that many men with LUTS do not consult a physician so they lack the medical attention that could alleviate their symptoms and worries. Only if a patient perceives his symptoms as a problem then will be consult a physician ${ }^{(5)}$. Different factors may be relevant in this process; it has been suggested that older men may accept chronic illness as part of ageing, which may also be true for LUTS ${ }^{(7)}$. Furthermore, some men may experience a stigma associated with their specific urinary symptoms such as dribbling and urgency, so it is difficult for them to discuss these with their doctors. Cunningham et al., $1996^{(6)}$ concluded from interviews that fear of cancer or surgery is yet another factor in a man's decision to consult a doctor. The man's perception of the care providers' ability to give relevant information or effective treatment is important, as is the patient's ability to cope with the $\operatorname{LUTS}^{(7)}$.

Several surveys of patient attitudes to $\mathrm{BPH}$ and its treatment have revealed that men are concerned about disease progression-over $60 \%$ in one survey worried about the future need for surgery and desire a drug therapy that will reduce the risks of complications and progression. Therefore a patient's concerns of the disease, and his subjective ranking of therapeutic priorities should be elicited, and the patient's subjective expectancies should be considered ${ }^{(3,8)}$.

Over the past decade there has been an increase in the number of men presenting with prostate disease. This had led to an expansion in the potential role of nurses in both assessment and management of these patients. The main goals of nursing care for patients with benign prostatic hyperplasia are based on three primary end points; accurate initial assessment, improved QOL through the alleviation of symptoms, and the prevention of long term complications ${ }^{(5)}$.

Nurses need to actively explore opportunities to promote health and to encourage and support men to make healthier lifestyle choices. To make appropriate health and lifestyle choices an individual must be provided with adequate information about his condition and its possible outcomes. He should also be offered opportunities to express his feelings, and explore treatment options. Nurses are in a key position to further their role as health teachers and they have unlimited opportunities to help patients manage their health. They work in settings where they have contact with large numbers of people of varied ages, cultural, and social backgrounds. In addition, they spend more time with patients than other team members; this contact provides the opportunity to develop trust, and to assess individual learning needs ${ }^{(7)}$.

\section{Aim of the Study}

This study aimed to describe patients' concerns regarding symptoms severity and treatment of benign prostate hypertrophy (BPH).

\section{Research Questions:}

The following questions were addressed in this study

1-What are patients' concerns regarding symptoms of $\mathrm{BPH}$ ?

2-What are patients' concerns regarding treatment of $\mathrm{BPH}$ ? 


\section{Materials and Method}

\section{Materials}

Design: A descriptive research design was used in this study.

Setting: This study was conducted at the Urologic Outpatient Department of the Health Insurance Hospital in Alexandria, Egypt.

Subjects: A convenience sample of 100 adult male patients diagnosed with BPH were recruited base on Epi info program. Men who were at least 45 years of age, with LUTS due to BPH were eligible to participate in the study. Patients were excluded if they reported a history of prostate or bladder cancer or if they had any associating chronic renal disorders lead to lower urinary tract symptoms.

Tool: A structured interview schedule was used in this study. It consisted of five parts; the first contained bio-sociodemographic data related to patient's age, marital status, education, and co morbidities. The second part included The International Prostate Symptom Score (IPSS) was used to determine the symptom level of LUTS $^{(9)}$. It is a validated symptom scoring instrument. It was developed in 1992 by the American Urological Association. It consists of 8 questions (7 questions concerning urinary symptoms and one question concerning quality of life). Questions concerning urinary symptoms include symptoms related to incomplete emptying, frequency, intermittency, urgency, weak stream, straining, and nocturia. A symptom index, ranging from 0 to 35 , was calculated by summing the scores of seven urinary symptoms where each question concerning urinary symptoms allows the patient to choose one out of six answers indicating increasing severity of the particular symptom. The answers are assigned points from 0 to 5 as the following values: never $=0$, hardly ever $=1$, less than half the time $=2$, about half the time $=3$, more than half the time $=4$ and almost always $=5$. The symptom index was categorized into four levels of severity; none (0) mild (1-7), moderate (8-19) and severe (20-35).

Participants were also asked about how they would feel if they had to spend the rest of their life with their current level of symptoms. This question was recommended by The International Scientific Committee, under the patronage of the World Health Organization (WHO) and the International Union against Cancer to assess the quality of life. The answers to this question ranged from "delighted" to "terrible" or 0 to 6 .

The third, the fourth and the fifth parts were developed by the researchers after reviewing the related literatures ${ }^{(3,6,8,12,13,17)}$. The third part involved 8 statements to assess patients' concerns about their initial BPH symptoms. Patients responses were checked on two choices (agree or disagree). The fourth part of tool included 9 statements that focused on what patients with enlarged prostate were told by their doctors at the time of diagnosis. The fifth part comprised with attributes of greatest importance to patients when considering medication for enlarged prostate.

The patients were asked to indicate the importance of medication they reported on a three-point Likert-type scale ranging from 'very important', 'some important' to 'not important'. The score assigned ranged from 3 to 1 with 3 for very important response and 1 for not important.

\section{Method}

Required permission for data collection was obtained from the director of the hospital. The study tool was initially translated into Arabic language and content validity of the tool was assessed by a panel of five experts in the field of medical surgical nursing for clarity, validity, and comprehensiveness of the tool. A pilot study with 10 participants, who were later excluded from the study, was conducted to 
determine the clarity and feasibility of the tool. The Cronbach alpha score for all parts of the tool ranged from 0.78 to 0.85 . Data were collected by researchers through individualized interviews with the patients at the Outpatient Urological Clinic of the hospital. These patients were given the chance to ask any question related to the study. All eligible patients took part in a 30min, face-to-face interviews based on the structured interview schedule. Data were collected over a period of 4 months from the beginning of June to the end of September 2012.

\section{Ethical considerations:}

The participants' rights were protected by explaining to the participants the purpose and significance of the study. Participants were reassured that their responses will be kept anonymous, and no remarks will be made to identify the client's identity. The client was informed that his participation in the study is voluntary and he can withdraw at any time, and that his withdrawal will not affect the care he receives at the hospital. Patients who agreed to participate in the study and met the inclusion criteria were asked to sign a written informed consent form.

\section{Statistical Analysis}

Data obtained from the study were analyzed using the Statistical Package for the Social Sciences version 15 (SPSS) computer program (SPSS Inc, Chicago, IL, USA). Descriptive and inferential statistics were performed on bio-sociodemographic data, the International Prostate Symptom Score (IPSS), as well as patients' concerns about symptoms severity and treatment of enlarged prostate. Chi square test was used to examine the relation between symptoms' severity and patient's concerns when seeking medical advice for symptoms of $\mathrm{BPH}$ as well as the relation between symptoms' severity and attributes of greatest importance to patients when considering medication for $\mathrm{BPH}$; values of $P<0.05$ were considered significant.

\section{Results}

\section{Characteristics of study participants:}

The mean age of participants was (61.65 years, $\mathrm{SD} \pm 4.61)$, the majority of the participants were married $(86 \%)$ whilst the highest portions for education (48\%) were holders secondary school certificates and higher education.

Table (1) shows Distribution of the studied patients according to international prostate symptom score (I-PSS). The results indicated that more than half of the patients (52\%) had an IPSS that ranged from 8-9, indicating moderate LUTS. Forty eight percent of patients had an IPSS of $\leq 7$, indicating mild LUTS. The mean IPSS was (8.03 scores and $\mathrm{SD} \pm 3.07$ ).

Table (2) shows the proportion of patients with $\mathrm{BPH}$ who recalled being told specific statements at the time of diagnosis. The findings revealed that $46 \%$ of patients were commonly told that "BPH is a normal part of aging" and "there are treatments available for symptoms relief". Large proportion of patients $(61 \%)$ recalled being told that "it is better to wait and see than to treat the condition from the time of diagnosis". Eleventh percent of patients recalled being told that "there was nothing to worry about and there is a treatment for underlying cause of enlarged prostate."

Table (3) reveals patients' concerns regarding symptoms of BPH. The main concerns first experienced by majority of the patients with symptoms of BEP when seeking medical advice were the impact of symptoms on social life and professional life, frustration, fear of cancer, and embarrassment caused by symptoms as reported by $81 \%, 70 \%, 67 \%, 64 \%$ and $61 \%$ of the patients, respectively.

Table (4) shows patients' concerns regarding treatment of $\mathrm{BPH}$. The findings indicated that up to $94 \%, 93 \%$, and $91 \%$ of 
the patients rated relieving symptoms within the first few weeks or months and reducing risk of surgery as very important attributes of medical therapy for benign enlarged prostate.

Table (5) reveals the relation between symptoms' severity and patient's concerns when seeking medical advice for symptoms of BPH. The results indicated that patients with moderate symptoms had higher percent of concerns with the impact of symptoms on social life and professional life, frustration, fear of cancer, and embarrassment caused by symptoms than those with mild symptoms .Moreover statistical significant differences were elicited (where $\mathrm{P}=0.036,0.001,0.016$, 0.001 , and 0.001 respectively).

Table (6) shows relation between symptoms' severity and attributes of greatest importance to patients when considering medication for $\mathrm{BPH}$. One can notice that there were no statistically significant relationships between symptoms severity and attributes of importance to patients when considering medication $(\mathrm{P}>$ $0.05)$.

Figure (1) displays distribution of IPSS severity by quality of life responses. The results indicated that men with moderate LUTS had a lesser quality of life score and their symptoms were more bothersome compared with those who had mild LUTS. More than one third ( $35 \%$ ) of patients with moderate LUTS were mostly dissatisfied and $33 \%$ of the patients were unhappy about their current urinary condition, compared with $27 \%$ and $18 \%$ of men with mild LUTS respectively.

\section{Discussion}

Understanding illness perceptions and incorporating them into health care is critical to effective treatment. Asking patients about how they view their illness gives health team the opportunity to identify and correct any inaccurate beliefs patients may have. Once a patient's illness perceptions are clearly laid out, a health team can try to direct those beliefs in a direction that is more compatible with treatment or better health outcomes ${ }^{(10)}$.

The study showed that the mean age of patients was above sixty years. In this context a previous study indicated that $\mathrm{BPH}$ is an extremely common condition among elderly men, reaching prevalence of approximately $70 \%$ in men over age 61 year and of greater than $90 \%$ in those over age 80 years $^{(8)}$.

The findings also revealed that less than half of the studied patients were commonly told that enlarged prostate is a normal part of aging and there are treatments available for symptoms. Large proportion of patients recalled being told that it is better to wait and see than to treat the condition from the time of diagnosis. Data from the Prostate Research on Behaviour and Education (PROBE) survey $^{(3)}$ and the Combination of Barkin et al studies $^{(11)}$ have provided a better understanding of preferences and satisfaction with $\mathrm{BPH}$ treatments, suggesting that patient and physician preferences may not always be aligned. In addition, prescribing practices differ somewhat between primary care physicians and urologists ${ }^{(12)}$. A similar study suggested that patients' views and beliefs and those of their physicians, may not always be in agreement. Improved physician-patient communication will help determine the best treatment options for patients with $\mathrm{BPH}$ and may ensure greater compliance and treatment success. Moreover, a better understanding of patient preferences should allow a more tailored treatment approach that may have benefits for improved patient compliance and therapeutic outcomes ${ }^{(13)}$.

When talking about patient's concerns of the symptoms, the results indicated that majority of subjects reported a fear of cancer as the reason for seeking medical advice. In this context a recent study of Petrie and Weinman (2012) revealed that patients who generally prepare themselves 
for an unfavorable diagnosis have higher levels of concern ${ }^{(14)}$. Other concerns first experienced by the patients were the impact of symptoms on social life and professional life, frustration and embarrassment caused by symptoms. These findings are supported by (Hassan, 2007) who reported that the severity of $\mathrm{BPH}$ symptoms interferes significantly with social activities of older adults $^{(15)}$. Where, LUTS as frequency of urination, urgency, urinary incontinence are causing embarrassment to them therefore, they prefer to stay home and avoid interaction with other people. In addition, Hassan, (2007) mentioned that in the Egyptian culture a person do not seek medical advice unless there are symptoms that interfere with his daily life activities. So, they do not seek medical help for screening purposes ${ }^{(15)}$. In this respect, Rosette (2006) study suggests that both, frequency of symptoms, and interference caused by symptoms are significant predictors of healthcare-seeking behavior $^{(16)}$.

On the other hand, Harkaway (2007) mentioned that many men do not seek medical advice for their symptoms because they recognize that their symptoms remit spontaneously and that they believe their symptoms are temporary, and they consider changes in urinary function to be part of the normal 'aging' process, or fail to report symptoms because of the fear of surgery, lack of awareness of effective medical therapies $^{(17)}$. Moreover, Ramesh and Kartheek (2009) reported that the patients could not consult their clinicians due to the fact that, they were not knowing that the symptoms were due to enlarged prostate ${ }^{(18)}$. This necessitates the importance of providing patient education to patients suffering from enlarged prostate. A previous research study of Rosenberg et al. (2013) indicated that what people believe about their illness may affect how they cope with it. It has been suggested that such beliefs stem from those commonly held within society $^{(19)}$.
It has been noticed that the main concerns of a large number of the studied patients, when taking treatment for enlarged prostate were the ability of medication to reduce the risk of surgery and relieve symptoms within few weeks or months. The interpretation may be that many patients are looking for the least treatment with lowest risk and they do not like the idea of surgery because they fear from side effects like urine leak, impotence or other complications that may arise from invasive procedures. This findings consistent with The American Urological Association (AUA) guideline that stress on the patient should play a central role in determining his need for treatment ${ }^{(20)}$.

Kaplan, (2007) reported that although acute urinary retention (AUR) is not life threatening, it is a serious QOL issue for patients. Consequently, prevention of AUR is desirable for men with EP, particularly those with risk factors such as moderate to severe LUTS and poor urinary-flow rates. Moreover, patients considered the insertion of a catheter for acute urinary retention to be more detrimental to their quality of life than surgery ${ }^{(21)}$.

Furthermore, Emberton et al. (2008) revealed that in general, reducing progression to surgery was favored over symptom relief regardless of whether patients were receiving $\alpha$-blocker or $5 \alpha$ reductase inhibitor (5ARI), although there was minor variation across countries ${ }^{(3)}$. Emberton (2010) study revealed that patients prefer therapies that affect longterm disease progression over those that provide short-term symptom relief. This preference may be underestimated by physicians, and there may be a general discord between patients' views and beliefs and those of their physician ${ }^{(12)}$. Therefore, it is vital that physicians assess and fully understand their patients' satisfaction with $\mathrm{BPH}$ treatment, their preferred treatment options and expectations. On the other hand, Kaplan and Naslund (2006) mentioned that despite the observation that avoiding 
surgery is a high priority among a large majority of enlarged prostate patients, only $18 \%$ were told a reduction in the risk of surgery could be achieved by medical therapy and only $40 \%$ of men with EP and moderate-to-severe symptoms have ever taken a prescription medication for their condition $^{(13)}$.

When patients were asked to describe if they had to live with their condition as it is now, how would they feel about it?; most men with moderate LUTS had a worse QOL score and their symptoms were more bothersome compared with men who had mild LUTS. In this respect, a number of studies using the International Prostate Symptom Score (IPSS) tool have documented that LUTS can have a significant impact on quality of life in men with $\mathrm{BPH}$, resulting in increased levels of bother and worry as well as interference with daily activities ${ }^{(22,23,24,25,26)}$. Men with moderate to severe symptoms have reported four to six times the degree of bother and interference with daily activities and twice the level of worry as men with mild symptoms $^{(23,27)}$. Hassan (2007) mentioned that QOL related to urinary symptoms was significantly inversely related to each of the specific urinary symptoms ${ }^{(15)}$.

In essence, the findings of this present study provide a valuable insight into the concerns of patients regarding $\mathrm{BPH}$ and its management. Moreover, it emphasizes the need for consideration of patient's illness perception during clinical decision-making. Previous studies revealed that, there are two important aspects to note: firstly, patients' beliefs about their condition are often at variance from those who are treating them. Medical staffs are usually unaware of patients' concerns about their condition as staffs rarely ask patients about their own ideas in clinical consultations. Secondly, patients' perceptions vary widely. Even patients with the same medical condition or injury can hold very disparate views of their illness ${ }^{(28,29,8,30)}$. In one survey, reduction in the risk of major urological complications and the need for surgery was of more importance to patients than improving symptoms and $\mathrm{QOL}^{(31)}$.

Therefore, choosing among the various treatment options to achieve management goals of BPH is a challenge to nurses caring for male patients with benign enlarged prostate and should be based not only on the best available evidence but also on patients' circumstances and personal choices. In addition, the benefits of treatment should be balanced against the risk of adverse events associated with therapy ${ }^{(32,33)}$.

\section{Conclusion}

The findings of this study highlight that $\mathrm{BPH}$ is a condition that concerns patients despite receiving treatment. Early in the presentation, patients' concern that their symptoms may indicate an underlying malignancy and may affect their social and professional life. When the disease is diagnosed as BPH, patients' concerns shift to reduce the need for surgery and relief symptoms within few weeks or months. This demonstrates a clear need for better patient education about BPH and its consequences. The results also indicated that patients with moderate LUTS had a worse quality of life score compared with patients who had mild LUTS. Better communication between nurses and other health team members and patients may facilitate improved understanding of patient concerns and selection of the most appropriate treatment.

\section{Recommendations}

The health team should be able to acknowledge patient's concerns, adequately informed the patient about the benefits and risks of the appropriate treatments, clarify the information, offer realistic hope, and avoid being over reassuring. Moreover, they must find out what the patient knows about the impact of the illness on his future and identify patient's coping strategies. 
Selecting an inappropriate treatment, or not including the patient's preference, will affect patients' treatment adherence and disease outcomes. Therefore, it is vital that health teams assess and fully understand their patients' satisfaction with $\mathrm{BPH}$ treatment, their preferred treatment options and expectations. 
Table (1): Distribution of the studied patients according to international prostate symptom score (I-PSS).

\begin{tabular}{|l|c|c||}
\hline & No. & \% \\
\hline Score & \multicolumn{2}{|c||}{$3.0-17.0$} \\
Range & \multicolumn{2}{|c|}{$8.03 \pm 3.07$} \\
Mean \pm SD & \multicolumn{2}{|c|}{8.0} \\
Median & 48 & 48.0 \\
Mild (IPSS 1-7) & 52 & 52.0 \\
Moderate (IPSS 8-19) & 0 & 0.0 \\
Severe (IPSS 20-35) & 0 \\
\hline
\end{tabular}

Table (2): Proportions of patients with BPH who recalled being told specific statements at the time of diagnosis (No.=100).

\begin{tabular}{|c|c|c|}
\hline *Statements told at the time of diagnosis & No. & \% \\
\hline What did the doctor say regarding diagnosis at the first visit? & & \\
\hline It is a normal part of aging & 46 & 46.0 \\
\hline Wait and see & 61 & 61.0 \\
\hline Treatment for symptoms & 46 & 46.0 \\
\hline Nothing to worry about & 11 & 11.0 \\
\hline There is treatment for the underlying causes of enlarged prostate. & 11 & 11.0 \\
\hline Medication can shrink prostate & 8 & 8.0 \\
\hline Surgery may be needed & 18 & 18.0 \\
\hline Medication can reduce the risk of surgery & 7 & 7.0 \\
\hline It could get worse & 27 & 27.0 \\
\hline
\end{tabular}

*: More than one response was allowed 
Table (3): Patients' concerns regarding symptoms of BPH (No.=100).

\begin{tabular}{||l|c|c|c|c|}
\hline \multirow{2}{*}{ Patients' concerns* } & \multicolumn{2}{|c|}{ Agree } & \multicolumn{2}{c|}{ Disagree } \\
\cline { 2 - 5 } & No. & $\%$ & No. & \% \\
\hline discomfort & 26 & 26.0 & 74 & 74.0 \\
\hline Interrupted Sleep & 9 & 9.0 & 91 & 91.0 \\
\hline Embarrassment of Symptoms & 61 & 61.0 & 39 & 39.0 \\
\hline Frustration of Symptoms & 67 & 67.0 & 33 & 33.0 \\
\hline Fear that it may be Cancer & 64 & 64.0 & 48 & 48.0 \\
\hline Impact of Symptoms on Work/Professional Life & 70 & 70.0 & 30 & 30.0 \\
\hline Impact of Symptoms on Social Life & 81 & 81.0 & 19 & 19.0 \\
\hline Affecting Marital Relationship & 50 & 50.0 & 50 & 50.0 \\
\hline
\end{tabular}

*: More than one response was allowed

Table (4): Patients' concerns regarding treatment of BPH (No.=100).

\begin{tabular}{|l|c|c|c|c|c|c||}
\hline \multirow{2}{*}{ Patients' concerns } & \multicolumn{2}{|c|}{ Not important } & \multicolumn{2}{c|}{ Some what } & \multicolumn{2}{c|}{$\begin{array}{c}\text { Very } \\
\text { important }\end{array}$} \\
\cline { 2 - 7 } & No. & $\%$ & No. & $\%$ & No. & $\%$ \\
\hline Cut the risk of surgery by 50\% & 3 & 3.0 & 6 & 6.0 & 91 & 91.0 \\
\hline $\begin{array}{l}\text { Provide symptom relief within the } \\
\text { first few weeks }\end{array}$ & 1 & 1.0 & 5 & 5.0 & 94 & 94.0 \\
\hline $\begin{array}{l}\text { Provide symptom relief within the } \\
\text { first few months }\end{array}$ & 1 & 1.0 & 6 & 6.0 & 93 & 93.0 \\
\hline $\begin{array}{l}\text { Reduce the size of the prostate and } \\
\text { maintain this reduction }\end{array}$ & 4 & 4.0 & 16 & 16.0 & 80 & 80.0 \\
\hline $\begin{array}{l}\text { Prevent further enlargement of the } \\
\text { prostate }\end{array}$ & 4 & 4.0 & 17 & 17.0 & 79 & 79.0 \\
\hline
\end{tabular}


Table (5): Relation between symptoms' severity and patient's concerns when seeking medical advice for symptoms of BPH (No.=100).

\begin{tabular}{||l|c|c|c|c|c||}
\hline \multirow{2}{*}{ Patient's concerns } & \multicolumn{3}{|c||}{ Symptoms' severity } & \multirow{2}{*}{} \\
\cline { 2 - 6 } & \multicolumn{2}{|c|}{$\begin{array}{c}\text { Mild symptoms } \\
\text { (n=48) }\end{array}$} & \multicolumn{2}{c||}{$\begin{array}{c}\text { Moderate } \\
\text { symptoms (n=52) }\end{array}$} & \multirow{2}{*}{ Test of sig. } \\
\cline { 2 - 6 } & No. & $\%$ & No. & \% & \\
\hline Discomfort & 13 & 27.1 & 13 & 25 & \multirow{2}{*}{$\chi^{2} \mathrm{p}=0.812$} \\
\hline Interrupted sleep & 3 & 6.3 & 6 & 11.5 & FEp $=0.305$ \\
\hline \begin{tabular}{|l} 
Embarrassment of symptoms \\
Frustration from symptoms
\end{tabular} & 19 & 39.6 & 42 & 80.8 & $\chi^{2} \mathrm{p}<0.001^{*}$ \\
\hline Fear that it may be cancer & 24 & 50 & 43 & 82.7 & $\chi^{2} \mathrm{p}<0.001^{*}$ \\
\hline $\begin{array}{l}\text { Impact symptoms } \\
\text { work/professional life }\end{array}$ & 21 & 43.8 & 43 & 82.7 & $\chi^{2} \mathrm{p}=0.016^{*}$ \\
\hline \begin{tabular}{l} 
Impact of symptoms on social life \\
\hline Affecting marital relationship
\end{tabular} & 29 & 60.4 & 41 & 78.8 & $\chi^{2} \mathrm{p}=0.001^{*}$ \\
\hline \hline
\end{tabular}

$\chi^{2} \mathrm{p}: \mathrm{p}$ value for Chi square test

FEp: $\mathrm{p}$ value for Fisher Exact test

*: Statistically significant at $\mathrm{p} \leq 0.05$

Table (6): Relation between symptoms severity and attributes of greatest importance to patients when considering medication for BPH (No.=100).

\begin{tabular}{|c|c|c|c|c|c|}
\hline \multirow{3}{*}{$\begin{array}{llr}\text { Attributes } & \text { of importance to } \\
\text { patients } & \text { when } & \text { considering } \\
\text { medication } & & \end{array}$} & \multicolumn{4}{|c|}{$\begin{array}{c}\text { \% Rating as ' very important' } \\
\text { Symptoms' severity }\end{array}$} & \multirow{3}{*}{ Test of sig. } \\
\hline & \multicolumn{2}{|c|}{$\begin{array}{c}\text { Mild symptoms } \\
(n=48)\end{array}$} & \multicolumn{2}{|c|}{\begin{tabular}{|c}
$\begin{array}{c}\text { Moderate symptoms } \\
(\mathrm{n}=52)\end{array}$ \\
\end{tabular}} & \\
\hline & No. & $\%$ & No. & $\%$ & \\
\hline Cut the risk of surgery by $50 \%$ & 41 & 85.4 & 50 & 96.2 & $\mathrm{FEp}=0.083$ \\
\hline $\begin{array}{l}\text { Provide symptom relief within the } \\
\text { first few weeks }\end{array}$ & 45 & 93.8 & 49 & 94.2 & $\mathrm{FEp}=1.000$ \\
\hline $\begin{array}{l}\text { Provide symptom relief within the } \\
\text { first few months }\end{array}$ & 45 & 93.8 & 48 & 92.3 & $\mathrm{FEp}=1.000$ \\
\hline $\begin{array}{l}\text { Reduce the size of the prostate and } \\
\text { maintain this reduction }\end{array}$ & 35 & 72.9 & 45 & 86.5 & $\chi^{2} \mathrm{p}=0.089$ \\
\hline $\begin{array}{l}\text { Prevent further enlargement of the } \\
\text { prostate }\end{array}$ & 40 & 83.3 & 39 & 75.0 & $\chi^{2} \mathrm{p}=0.307$ \\
\hline
\end{tabular}

$\chi^{2} \mathrm{p}: \mathrm{p}$ value for Chi square test

FEp: $\mathrm{p}$ value for Fisher Exact test

*: Statistically significant at $\mathrm{p} \leq 0.05$ 


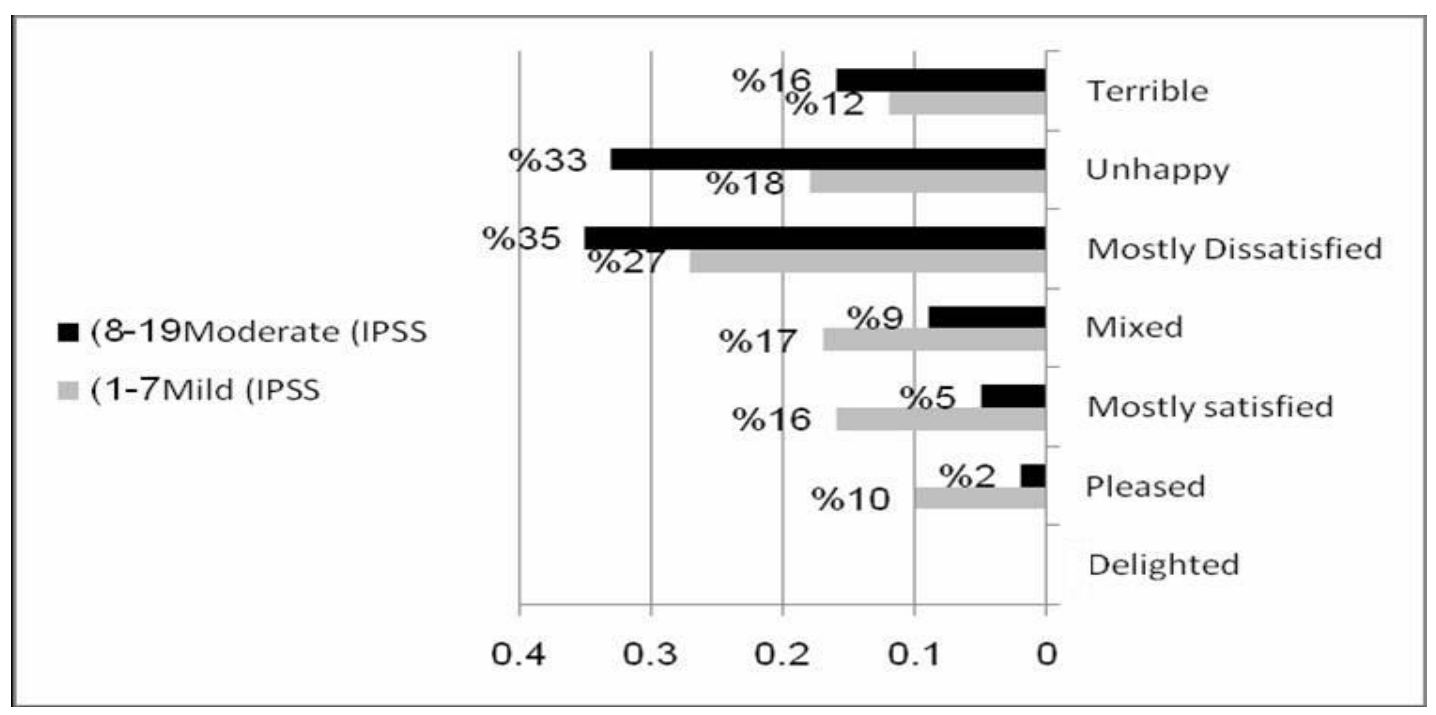

Figure 5: Distribution of the International Prostate Symptom Score (IPSS) severity by quality of life responses 


\section{References}

1. Naslund M.J, Gilsenan A.W, Midkiff K.D, Bown A, Wolford E.T\& Wang A. Prevalence of lower urinary tract symptoms and prostate enlargement in the primary care setting. International Journal of Clinical Practice 2007; 61(9): 1437-45.

2. Levi A. Benign Prostate hypertrophy. Medscape (2011). http://emedicine.Medscape.com/article/4 $\underline{37359}$.

3. Emberton M, Marberger M, \& Rosette J. Understanding patient and physician perceptions of benign prostatic hyperplasia in Europe: The prostate Research on Behaviour and Education (PROBE) Survey. International Journal of Clinical Practice 2008; 62(1): 18-26.

4. Lee N.G, Xue H, Lerner L. Trends and Attitudes in Surgical Management of Benign Prostatic Hyperplasia. Canadian Journal Urology 2012; 1(2):6170-75.

5. Hongjing W., Yuan L., Yanhua L., Yingsheng C., and Ping Y., Healthrelated behaviours and family support and clinical symptoms of BPH: a pilot study from China. International Journal of Urological Nursing 2013; (1):9-16.

6. Cunningham B, Allbutt H, Garraway,W and Lee A.J. Perceptions of urinary symptoms and health-care -seeking behavior amongst men aged 40-79 years. British Journal of General Practice. 1996; 46(407): 349-52.

7. Wolters R, Wensing M and Van Weel C. Lower urinary tract symptoms: social influence is more important than symptoms in seeking medical care. BJU International 2002; 90(7): 655-61.

8. Emberton M, Martorana G. BPH: social impact and patient's perspective. European Urology Supplements 2006; 5(20): 991-96.

9. International prostate symptom score. Available
http://www.usrf.org/questionnaires/AU SymtomScore.html.

10. Nauert R. Perception of illness influences outcome. Journal of Association for Psychological Science 2012; 30(10): 23-31.

11. Barkin, J, Roehrborn, C, Siami, P. Effect of dutasteride, tamsulosin and the combination on patient-reported quality of life and treatment satisfaction in men with moderate-to-severe BPH: 2-year data from the CombAT trial. BJU International 2009; 103(7): 919-26.

12. Emberton M. Medical treatment of benign prostatic hyperplasia: physician and patient preferences and satisfaction. International Journal of Clinical Practice 2010; 64(10):1425-35.

13. Kaplan S, Naslund M. Public, patient, and professional attitudes towards the diagnosis and treatment of enlarged prostate: a landmark national US survey. International Journal of Clinical Practice 2006; 60 (10):1157-65.

14. Petrie K.J, Weinman J. Patients' perceptions of their illness: the dynamo of volition in health care. Current Directions in Psychological Science 2012; 21(7): 60-5.

15. Hassan B. (2007). Quality of life of elderly patients with benign prostatic hyperplasia. Unpublished Doctoral Thesis. Faculty of Nursing Alexandria University.

16. Rosette J. Optimizing assessment and treatment decisions for men with $\mathrm{BPH}$. European urology supplements 2006; 5(12): 710-15.

17. Harkaway R. What are the Views of Patients and Urologists on Benign Prostatic Hyperplasia and Its Management? European urology supplements 2007; 6(6): 454-59.

18. Ramesh A, \& Kartheek S. Study of impact of patient education on health 
related quality of life in benign prostate hyperplasia (BPH) patients in A south Indian Teaching Hospital. Asian Journal of Pharmaceutical and Clinical Research 2009; 2(4): 97-103.

19. Rosenberg MT, Staskin D, Riley J, Sant $\mathrm{G}$, and Miner M. The evaluation and treatment of prostate-related LUTS in the primary care setting: the next STEP. Current Urology Reports Journal. 2013; 14(6):595-605.

20. Rosenberg M.T, Staskin D.R, Kaplan S.A, MacDiarmid S.A, and Newman D.K. Practical Guidelines for the Treatment of Enlarged Prostate in the Primary Care Setting. International Journal of Clinical Practice 2007; 61(9): 1535-46.

21. Kaplan S. IPSS Quality of Life Question: A possible indicator of depression among patients with lower urinary tract symptoms. The Journal of Urology 2012; 188 (5):1847-48.

22. Warren K, Burden H, Abrams P. Lower urinary tract symptom: still too much focus on the prostate? Current opinion in urology 2014 Jan; 24(1):3-9.

23. Kaplen S. Sleep analysis of patients with nocturia and benign prostatic obstruction. The Journal of Urology 2013; 189(6):2212-13.

24. King C, Hinds P. Quality of life from nursing and patient perspectives.2nd ed. Canada: Jones and Bartlett, 2003; 3-11, 93-112.

25. Mandal S., Massey M. and Goel A .Cannon-ball bladder stones: a complication of prolonged recumbence and benign prostate enlargement.
International Journal of Urological Nursing 2014; 8 (1): 15-18.

26. Dirksen S, Lewis S, Heitkemper $M$. Companion to Medical-surgical nursing. Assessment and Management of clinical problems. 8th ed. USA: Elsevier, Inc., 2011; 69-75.

27. Kirby et al. Fast facts: benign prostatic hyperplasia International Journal of Urological Nursing 2013; 7(2): 117-20.

28. Petrie K.J, Jago L.A, \& Devcich D.A. The role of illness perceptions in patients with medical conditions. Current Opinion in Psychiatry 2007; 20(2): 163 167.

29. Prosenjit G, Poole J, Nightingale P, \& Robertson A. Perceptions of illness and their impact on sickness absence. Occupational Medicine 2009; 59(4): 550-5.

30. Petersen Kapoor A. Benign prostate hyperplasia $(\mathrm{BPH})$ management in the primary care setting. The Canadian Journal of Urology 2012; 19 (supplement): 10-17.

31. Harkaway RC, Issa M. Medical and minimally invasive therapies for the treatment of benign prostatic hyperplasia. Prostate Cancer and Prostatic Diseases 2006; 9(3): 204-214.

32. Barkin J. Benign prostatic hyperplasia and lower urinary tract symptoms: evidence and approaches for best case management. The Canadian Journal of Urology.2011; 18 (supplement 1):14-19.

33. Elterman DS, Barkin J, Kaplan SA. Optimizing the management of benign prostatic hyperplasia. Therapeutic Advances in Urology 2012; 4(2): 77-83. 